Revista Brasileira de Agricultura Irrigada v.13, nº.6, p. 3741 - 3748, 2019

ISSN 1982-7679 (On-line)

Fortaleza, CE, INOVAGRI - http://www.inovagri.org.br

DOI: $10.7127 /$ rbai.v13n6001149

Protocolo 1149.19 - 21/04/2020 Aprovado em 11/05/2020

\title{
MODELO DE SIMULAÇÃO PARA AVALIAR O IMPACTO DAS CONDIÇÕES DO CLIMA E DA PLANTA NA LÂMINA IRRIGADA
}

\author{
Élvis Da Silva Alves ${ }^{1}$, Lineu Neiva Rodrigues ${ }^{2}$, Douglas Ramos Lorena ${ }^{4}$, Diego Bispo Dos Santos \\ Farias $^{5}$
}

\begin{abstract}
RESUMO
O aumento da competição pelo uso de recursos hídricos pode ocasionar conflitos pelo uso da água e, manejar a irrigação com eficiência é, sem dúvidas, uma das principais soluções para a gestão correta dos recursos hídricos. A utilização de ferramentas computacionais pode simplificar o gerenciamento dos recursos hídricos. O Modelo de Simulação de Estratégias de Irrigação (MSEI) detalha o manejo da irrigação de forma simulada para um grande intervalo de tempo, proporcionando informações que podem servir de base na tomada de decisão para os anos posteriores. Desta forma, esse trabalho objetivou conhecer a influência de algumas variáveis do clima e da planta na lâmina total de irrigação através do MSEI. Para realização do estudo, foram adotadas informações dos solos e das condições climatológicas do Cerrado brasileiro. Considerou-se 31 anos de precipitação e evapotranspiração de referência. Como variável de estudo, adotou-se a profundidade efetiva do sistema radicular. Através das simulações geradas pelo MSEI foi possível identificar que a diminuição do sistema radicular máxima da cultura, no momento do manejo, resultou em maiores lâminas totais de irrigação, com valores superiores à de $90 \%$, nos anos do estudo. Nos anos em que os eventos de precipitação acumulada foram maiores, a demanda por irrigação foi menor. Enquanto que nos anos de menores índices de precipitação, a demanda aumentou para todos as profundidades do sistema radicular. Nos anos de menores índices de precipitação, a demanda aumentou, para todas as profundidades do sistema radicular. O MSEI permitiu a simulação de diferentes cenários da lâmina de irrigação com agilidade e confiança.
\end{abstract}

Palavras-chave: Manejo de irrigação, MSEI, modelagem.

\footnotetext{
${ }^{1}$ Engenheiro Agrônomo, Doutorando em Engenharia Agrícola, Universidade Federal de Viçosa, Viçosa, MG. Fone (61) 98271-1768, e-mail:elvistv@gmail.com

${ }^{2}$ Pesquisador, Embrapa Cerrados, Caixa Postal 08223, CEP 73310-970, Planaltina, DF. Fone (61) 3388-9959. e-mail: lineu.rodrigues@embrapa.br

${ }^{3}$ Professor, Universidade Federal de Viçosa, Viçosa, MG.

${ }^{4}$ Engenheiro Agrônomo, Doutorando em Agronomia, Universidade de Brasília, Brasília, DF. Fone (61) 99606-2414, email: douglasramoslorena@gmail.com

${ }^{5}$ Engenheiro Agrônomo, Mestrando em Engenharia Agrícola, Universidade Federal de Viçosa, Viçosa, MG. Fone (31) 99816-2080, e-mail: diegotrust@gmail.com
} 


\title{
SIMULATION MODEL TO EVALUATE THE IMPACT OF CLIMATE AND PLANT CONDITIONS ON THE IRRIGATED DEPTH
}

\begin{abstract}
Increased competition for the use of water resources can lead to conflicts over the use of water and, managing irrigation efficiently is undoubtedly one of the main solutions for the correct management of water resources. The use of computational tools can simplify the management of water resources. The Simulation Model for Irrigation Strategies (MSEI) details the management of irrigation in a simulated way over a long period of time, providing information that can serve as a basis in decision making for later years. In this way, this work aimed to know the influence of some climate and plant variables on the total irrigation depth through the MSEI. To conduct the study, they were adopted information of soil and climatic conditions of the Brazilian Cerrado. It was considered 31 years of reference precipitation and evapotranspiration. As a study variable, the effective depth of the root system was adopted. In the years when accumulated precipitation events were higher, the demand for irrigation was lower. While in the years of lower rainfall, the demand increased for all depths of the root system. In the years of lower precipitation rates, demand increased for all depths of the root system. The MSEI allowed the simulation of different scenarios of the irrigation blade with agility and confidence.
\end{abstract}

Keywords: Irrigation management, MSEI, modeling.

\section{INTRODUÇÃO}

Apesar do aumento da produção mundial de alimentos, a garantia de um acesso igualitário a comida, que assegure quantidades suficientes de alimento para manter níveis básicos de saúde, não é assegurada para atender uma população mundial que chegará a 10 bilhões de habitantes em 2050 (FAO, 2017; NATIONS, 2014; RAMANKUTTY et al., 2018).

Aumentar a produção de alimentos sem a abertura de novas áreas só será possível com a intensificação nas áreas já cultivadas e com o aprimoramento das tecnologias, principalmente da irrigação, que é a principal usuária de recursos hídricos. Porém, a irrigação pode aumentar a produtividade da água e melhorar a eficiência de aplicação (AHMADI et al., 2010; BATTILANI; LETTERIO; CHIARI, 2014; JENSEN et al., 2014) o que tornará os sistemas irrigados mais sustentáveis. Para Rodrigues e Domingues (2017), a produção sustentável, através da agricultura irrigada, exige o uso contínuo da água, visando sempre a sua eficiência.

O milho é uma das principais culturas produzidas no Brasil e no mundo. Além de ser um dos principais componentes da alimentação humana, também é a base principal da alimentação animal. $\mathrm{Na}$ safra 2019/2020 o Brasil colheu 101,90 milhões de toneladas, aumento de $1,8 \%$ em relação à safra 2018/19 (CONAB, 2020). Devido a sua importância, faz-se necessário o aumento da produtividade e produção. Apenas com o aperfeiçoamento das técnicas produtivas, como o usa da irrigação é possível atingir cenários melhores. Manejar corretamente a irrigação não é tarefa fácil, principalmente quando se objetiva trabalhar com precisão. Rodrigues e Moreira (2015) afirmaram que a complexibilidade dos ambientes produtivos, devido à quantidade de dados cada vez maior, juntamente com a enigmática relação dos fatores ligados a tomadas de decisão, agregados ao clima, solo, planta e sistema de irrigação, complexificam no momento do planejamento e tomada de decisão.

Diversos modelos computacionais surgiram com o advento da programação, trazendo consigo a facilidade de cálculos antes complexos. Recentemente, modelos e ferramentas foram utilizados para facilitar o agendamento da irrigação como, por exemplo, Ritchie (KODUR, 2017), MSEI 
(RODRIGUES; MOREIRA, 2015), CROPWAT (AUGUSTIN et al., 2015), SWAT (MAIER; DIETRICH, 2016), SIMERAW (MANCOSU et al., 2016), AquaCrop (LINKER et al., 2016; PAREDES et al., 2015), DAISY (SEIDEL et al., 2016), FAO Kc Dual (ANDERSON et al., 2017), além de modelos matemáticos simples (NETO et al., 2019). É possível realizar diversas simulações utilizando esses modelos, identificando detalhadamente a influência de algumas variáveis de forma isolada em uma variável determinante. Um desses modelos é o Modelo de Simulação de Estratégias de Irrigação-MSEI (RODRIGUES; MOREIRA, 2015). Portanto, esse trabalho objetivou conhecer a influência da profundidade do sistema radicular e da precipitação acumulada na lâmina total de irrigação através do MSEI.

\section{MATERIAL E MÉTODOS}

\section{Informações sobre o MSEI}

Segundo com Rodrigues e Moreira (2015), o modelo permite ao usuário escolher diversas opções de configuração do sistema, podendo modificar informações de solo, regime de irrigação (totalmente irrigado ou sequeiro), tipo de manejo (irrigação total ou com déficits percentuais), manejo para oito culturas, escolher entre a opção data de plantio definida ou semeadura para todos os dias do ano. Para a cultura, pode ser modificado a duração do ciclo, o número de dias para atingir a emergência, o coeficiente de cultivo (Kc), o coeficiente de estresse hídrico (Ks), o coeficiente de rendimento da cultura (Ky). Além disso, pode-se alternar entre uma função de crescimento radicular linear ou sigmoidal, o tamanho inicial e final da raiz e o percentual de crescimento radicular. Ainda pode ser definido o tipo de estratégia de manejo de irrigação, podendo ser intervalo de tempo de irrigação fixo, lâmina de irrigação fixa ou manejo do déficit de água no solo, inserindo manualmente um fator de disponibilidade do solo (f).

$\mathrm{Na}$ Figura 1 foi possível observar a tela inicial do MSEI, contendo as abas das configurações gerais, solo, cultura, irrigação e clima.

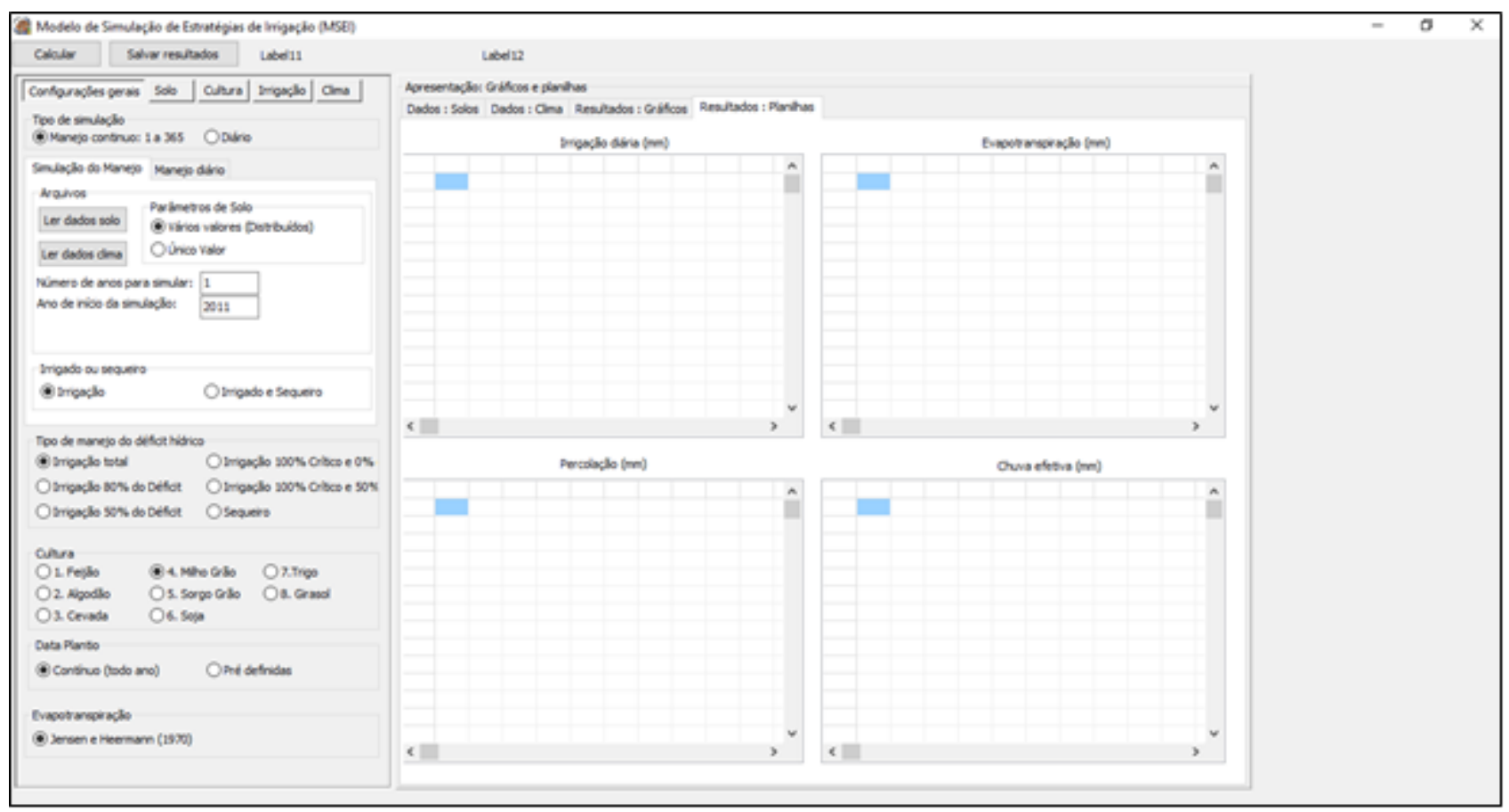

Figura 1. Tela representativa do software MSEI mostrando as abas de acesso e inserção dos dados. 


\section{Dados de entrada do MSEI}

Para realização da simulação através do MSEI (Figura 1), foram adotados valores baseando-se nos solos e nas condições climatológicas do Cerrado brasileiro. Considerou-se 31 anos (1980-2011) de precipitação e evapotranspiração de referência
(ETo). Esses dados foram obtidos na estação meteorológica da Embrapa Cerrados.

A evapotranspiração de referência $\left(\mathrm{ET}_{0}\right)$ foi calculada pela equação FAO-Penman Monteith (ALLEN et al., 1998).

Na Tabela 1 estão apresentados os dados de solo e da cultura, utilizados na simulação da lâmina de irrigação total por ciclo.

Tabela 1. Valores de capacidade de campo (CC), ponto de murcha permanente (PMP), densidade global do solo (Ds), profundidade máxima do sistema radicular (Z), fator de disponibilidade hídrica (f), data de semeadura e duração do ciclo utilizados como entrada do modelo para realização da simulação.

\begin{tabular}{|c|c|c|c|c|c|}
\hline $\begin{array}{c}\mathrm{CC} \quad \mathrm{PMP} \\
(\%)\end{array}$ & Ds $\left(\mathrm{g} \mathrm{cm}^{-3}\right)$ & $\mathrm{Z}(\mathrm{cm})$ & (f) & $\begin{array}{c}\text { Data de } \\
\text { semeadura }\end{array}$ & $\begin{array}{c}\text { Duração do ciclo } \\
\text { (dias) }\end{array}$ \\
\hline 26,8 & 1,05 & 60 & 0,5 & $10 / 10$ & 140 \\
\hline
\end{tabular}

\section{Estrutura da simulação}

Com a utilização do MSEI foi realizada a simulação da demanda de água da cultura do milho destinado à produção de grãos (milho grão) nas condições do Cerrado brasileiro, especificamente da região onde encontra-se a Embrapa Cerrados, Planaltina - DF.

Inicialmente foi inserido os arquivos .txt referente as informações do solo (CC, PMP e Ds). Em seguida foram inseridos os dados do clima (chuva e ETo) também em arquivo txt. Depois foi selecionado o regime hídrico adotado na simulação, podendo escolher entre irrigado ou irrigado + sequeiro. Por sim, foi selecionada a cultura a ser estudada. Após apertar no botão calcular, como se ver no lado superior esquerdo da Figura 1, programa gera autenticamente os arquivos de saída. Dentro do MSEI, a modificação realizada foi a profundidade máxima do sistema radicular $(40,50$ e $60 \mathrm{~cm})$ para a cultura do milho grão.

\section{RESULTADOS E DISCUSSÃO}

\section{Crescimento do sistema radicular da cultura do milho grão utilizado na simulação}

Observou-se que, a depender da profundidade máxima adotada no modelo, o dia após o plantio (DAP) em que a raiz atinge o valor máximo foi diferente (Figura 2A). $\mathrm{Na}$ profundidade máxima de $40 \mathrm{~cm}$, atingiu-se o valor máximo aos 96 dias após o plantio (DAP). Para as profundidades de 50 e $60 \mathrm{~cm}$ foi aos 99 e 101 DAP, respectivamente.

Quanto maior a profundidade do sistema radicular maior será a necessidade hídrica da cultura, e consequentemente a lâmina de irrigação será maior. Curtis et al. (1990) afirmaram que a maior expansão do sistema radicular possibilitou a exploração de maior volume de solo, aumentando a taxa de absorção de água. 

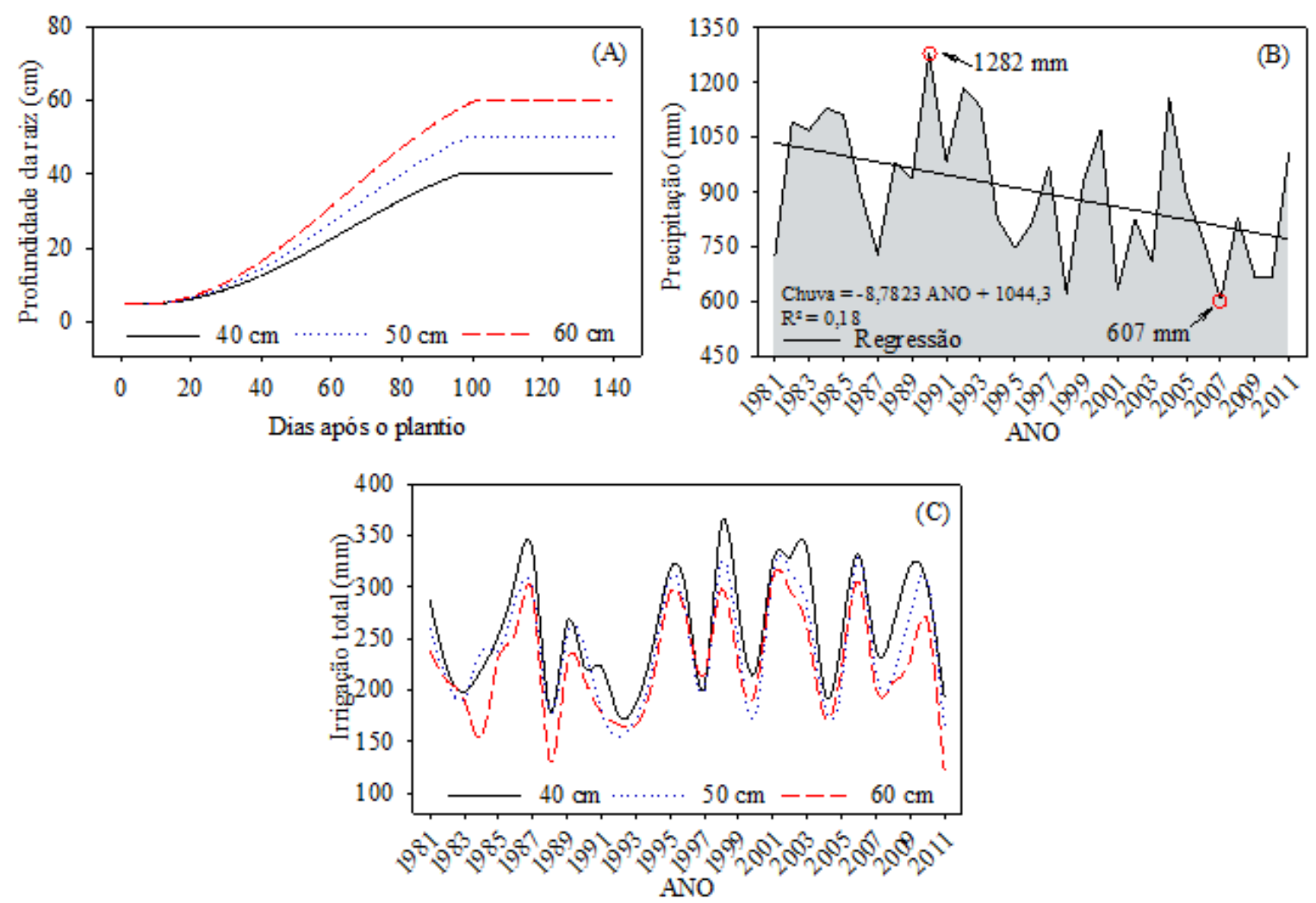

Figura 2. Crescimento do sistema radicular da cultura do milho grão, para as todas as condições de estudo (A); comportamento da precipitação (B); comportamento da lâmina de irrigação acumulada no ciclo para as todas as condições de estudo, durante os 31 anos da simulação (C).

\section{Comportamento da precipitação durante o estudo}

A variação na precipitação entre o maior e menor valor acumulado foi de $47,40 \%$, tendo 1990 como o ano de maior quantidade de chuva e 2007 o que apresentou menor total de precipitação (período de 1981 a 2011), conforme o destaque em vermelho (Figura 2B). O valor médio da $\Sigma$ Pac durante os 31 anos foi de $904 \mathrm{~mm}$.

Apesar do baixo valor do coeficiente de determinação, a precipitação apresentou queda durante o período de estudo (Figura 2B), demonstrando a diminuição dos eventos de precipitação no período de 31 anos. Para a agricultura, a diminuição das chuvas é algo ruim, já que maior parte das áreas do Brasil são cultivas no regime de sequeiro. De acordo com dados da ANA (2017), apenas 6,95 Mha da área agricultável do Brasil é irrigada. Tanto o aumento do número de dias consecutivos sem ocorrência de precipitação pluvial (veranicos) quanto a diminuição do número de dias com precipitação, durante o ciclo da cultura, influenciam diretamente na queda de rendimento da cultura da soja (RADIN; SCHÖNHOFEN; TAZZO, 2018).

\section{Impacto da profundidade da raiz de plantas de milho na lâmina total de irrigação}

A lâmina de irrigação total no ciclo aumentou com a diminuição da profundidade do sistema radicular (Figura 2C). Este comportamento justifica-se pela interferência direta no déficit máximo permissível da água no solo (MAD), decorrente do menor armazenamento da água e aumento da frequência de irrigação. Como a lâmina de água no solo depende também da evapotranspiração atual, precipitação, lâmina da irrigação e da quantidade de água armazenamento no solo no dia anterior, o MAD tende a ser apenas uma parcela da lâmina.

Considerando o sistema radicular de 60 cm como padrão no manejo da irrigação para a 
cultura do milho grão, quando assumiu a profundidade de $50 \mathrm{~cm}$ para o manejo durante todo ciclo da cultura, notou-se um aumento de 6,65\% na lâmina total acumulada nos 31 anos. Quando a profundidade adotada foi de $40 \mathrm{~cm}$, esse aumento foi de $13,85 \%$.

\section{Impacto da precipitação na lâmina total de irrigação}

A Figura 3 mostra a relação existente entre os eventos de precipitação e a lâmina de irrigação acumulada, considerando diferentes profundidades da raiz no manejo da irrigação.

Nos anos com maior precipitação acumulada a demanda por irrigação tende a ser menor, assim como nos anos com menor precipitação ocorre o inverso para todos as profundidades do sistema radicular.

Ainda é possível observar que, mesmo seguindo uma tendência, há variação da demanda ano a ano, mesmo com elevados valores de precipitação necessitam de lâminas maiores de irrigação. As áreas em destaque na Figura 3 reforçam esta observação.

Quando os eventos de precipitação forem mal distribuídos (DALLACORT et 2011), a irrigação será sempre necessária para garantia de safra e alta rentabilidade da cultura em questão (FIGUEIREDO et al., 2008).

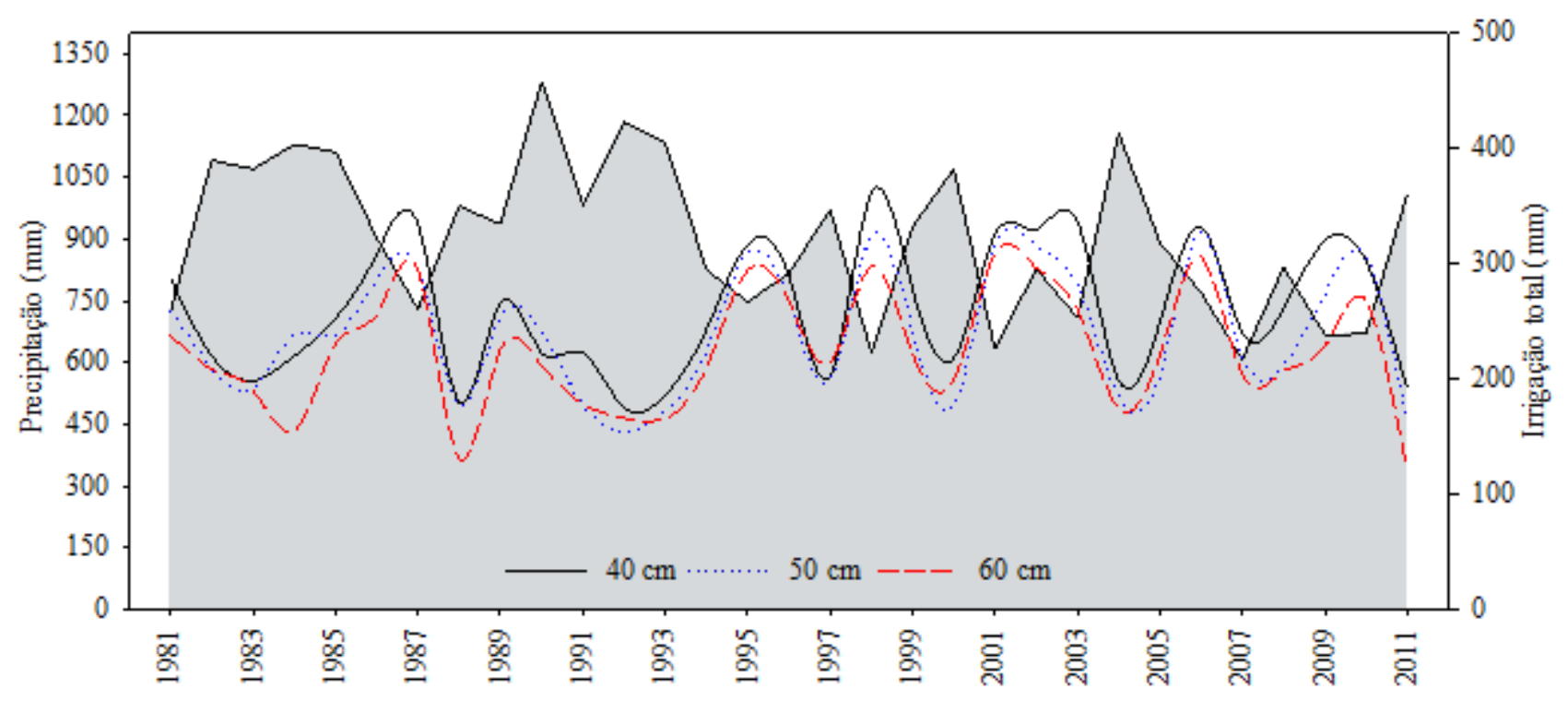

Figura 3. Irrigação total para diferentes profundidades do sistema radicular da cultura do milho grão e precipitação, durante 31 anos de simulação.

Considerando os 31 anos de estudo, quando se analisou a precipitação acumulada durante o ciclo da cultura do milho frente à lâmina demandada, observou-se que mesmo no ano de maior precipitação (506\% maior que a lâmina total na profundidade de $60 \mathrm{~cm}$ ), foi necessária a aplicação de uma lâmina de 211 mm para suprir a demanda da cultura, reforçando a idéia da influência da má distribuição temporal das chuvas e a importância da irrigação.

Para Saadi et al. (2015), as mudanças climáticas tendem a ser um dos grandes influenciadores da agricultura. Os autores ainda destacaram a necessidade do conhecimento de possíveis cenários futuros para potencializar a tomada de decisão, que devem ser integrados em um sistema complexo que liga as relações biofísicas, socioeconômicas e questões de política.

O MSEI surge como uma ferramenta que permite o conhecimento dos cenários futuros, além de possibilitar o manejo da irrigação de forma diária, facilitando o planejamento agrícola e a tomada de decisão.

\section{CONCLUSÕES}

O Modelo de Simulação de Estratégias de Irrigação (MSEI) permite a simulação de diferentes cenários da lâmina de irrigação para 
a cultura do milho grão, com agilidade e confiança;

A diminuição do sistema radicular máximo da cultura do milho, no momento do manejo, resultou em maiores lâminas totais de irrigação;

Nos anos em que os eventos de precipitação acumulada foram maiores, a demanda por irrigação foi menor;

Nos anos de menores índices de precipitação, a demanda aumentou, para todas as profundidades do sistema radicular.

\section{AGRADECIMENTOS}

À Fundação de Apoio à Pesquisa do Distrito Federal (FAPDF), pelo apoio financeiro; ao Conselho Nacional de Desenvolvimento Científico e Tecnológico (CNPq), pela concessão da bolsa do primeiro autor; e a Embrapa Cerrados, por disponibilizar dos dados utilizados neste estudo.

\section{REFERÊNCIAS BIBLIOGRÁFICAS}

AHMADI, S. H.; ANDERSEN, M. N.; PLAUBORG, F.; POULSEN, R. T.; JENSEN, C. R.; SEPASKHAH, A. R.; HANSEN, S. Effects of irrigation strategies and soils on field grown potatoes: Yield and water productivity. Agricultural Water Management, v. 97, n. 11, p. 1923-1930, 2010.

https://doi.org/10.1016/j.agwat.2010.07.007

ALLEN, R. G.; PEREIRA, L. S.; RAES, D.; SMITH, M. Crop Evapotranspiration Guidelines for Computing Crop Water Requirements. FAO Irrigation and drainage paper 56. Roma, Italy: Food and Agriculture Organization of the United Nations, 1998.

ANA (ED.). Atlas Irrigação: Uso da Água na Agricultura Irrigada. Brasília, DF: ANA, 2017.
AUGUSTIN, L. K.; YAGOOB, A. H.; KIRUI, W. K.; PEILING, Y. Optimal Irrigation Scheduling for Summer Maize Crop: Based on GIS and CROPWAT Application in Hetao District; Inner Mongolia Autonomous Region, China. Journal of Biology, p. 10, 2015.

BATTILANI, A.; LETTERIO, T.; CHIARI, G. AquaCrop model calibration and validation for processing tomato crop in a sub-humid climate. In XIII International Symposium on Processing Tomato 1081. Anais 2014. p. 167174.

https://doi.org/10.17660/ActaHortic.2015.108 1.19

CONAB - Companhia Nacional de Abastecimento. Safra Brasileira de Grãos. Disponível em: <https://www.conab.gov.br/infoagro/safras/graos〉. Acesso em: 05 abr. 2020.

CURTIS, P. S.; BALDUMAN, L. M.; DRAKE, B. G.; WHIGHAM, D. F. Elevated Atmospheric $\mathrm{CO} 2$ Effects on Belowground Processes in $\mathrm{C} 3$ and $\mathrm{C} 4$ Estuarine Marsh Communities. Ecology, v. 71, n. 5, p. 20012006, 1990. https://doi.org/10.2307/1937608

DALLACORT, R.; MARTINS, J. A.; INOUE, M. H.; FREITAS, P. S. L. D.; COLETTI, A. J. Distribuição das chuvas no município de Tangará da Serra, médio norte do Estado de Mato Grosso, Brasil. Acta Scientiarum. Agronomy, v. 33, n. 2, 2011. https://doi.org/10.4025/actasciagron.v33i2.583 8

FAO (ED.). Building resilience for food and food security. Rome: FAO, 2017.

FIGUEIREDO, M. G. D.; PITELLI, M. M.; FRIZZONE, J. A.; REZENDE, R. Lâmina ótima de irrigação para o feijoeiro considerando restrição de terra e aversão ao risco. Acta Scientiarum. Agronomy, v. 29, n. 5, p. 593-598, 12 fev. 2008. https://doi.org/10.4025/actasciagron.v29i5.733 
JENSEN, C. R.; ØRUM, J. E.; PEDERSEN, S. M.; ANDERSEN, M. N.; PLAUBORG, F.; LIU, F.; JACOBSEN, S. E. A short overview of measures for securing water resources for irrigated crop production. Journal of agronomy and crop science, v. 200, n. 5, p. 333-343, 2014. https://doi.org/10.1111/jac.12067

LINKER, R.; IOSLOVICH, I.; SYLAIOS, G.; PLAUBORG, F.; BATTILANI, A. Optimal model-based deficit irrigation scheduling using AquaCrop: A simulation study with cotton, potato and tomato. Agricultural Water Management, v. 163, p. 236-243, 2016.

https://doi.org/10.1016/j.agwat.2015.09.011

MAIER, N.; DIETRICH, J. Using SWAT for strategic planning of basin scale irrigation control policies: a case study from a humid region in northern Germany. Water Resources Management, v. 30, n. 9, p. 32853298, 2016. https://doi.org/10.1007/s11269016-1348-0

MANCOSU, N.; SPANO, D.; ORANG, M.; SARRESHTEH, S.; SNYDER, R. L. SIMETAW\# - a Model for Agricultural Water Demand Planning. Water Resources Management, v. 30, n. 2, p. 541-557, 2016. http://dx.doi.org/10.1007/s11269-015-1176-7

NATIONS, U. World Population Prospects, 2017 Revision; 2017. New York: United Nations, 2014. RADIN, B.; SCHÖNHOFEN, A.; TAZZO, I. F. Impacto da quantidade e frequência de chuva no rendimento da soja. Agrometeoros, v. 25, n. 1, 2018. http://dx.doi.org/10.31062/agrom.v25i1.26263

RAMANKUTTY, N.; MEHRABI, WAHA, K.; JARVIS, L.; KREMEN, C.; Z.;
HERRERO, M.; RIESEBERG, L. H. Trends in Global Agricultural Land Use: Implications for Environmental Health and Food Security. Annual Review of Plant Biology, v. 69, n. 1, p. 789-815, 2018. https://doi.org/10.1146/annurev-arplant042817-040256

RODRIGUES, L. N.; DOMINGUES, A. F. Agricultura Irrigada: desafios e oportunidades para o desenvolvimento sustentável. Brasília: INOVAGRI, 2017.

RODRIGUES, L. N.; MOREIRA, J. M. M. A. P. Desenvolvimento de um modelo de simulação de estratégias de irrigação. In: III INOVAGRI INTERNATIONAL MEETING. Fortaleza, Ceará, Brasil: INOVAGRI/INCTEI, 2015. Anais do III Inovagri International Meeting - 2015. http://dx.doi.org/10.12702/iii.inovagri.2015a197

SAADI, S.; TODOROVIC, M.; TANASIJEVIC, L.; PEREIRA, L. S.; PIZZIGALLI, C.; LIONELLO, P. Climate change and Mediterranean agriculture: Impacts on winter wheat and tomato crop evapotranspiration, irrigation requirements and yield. Agricultural Water Management, Agricultural Water Management: Priorities and Challenges. v. 147, p. 103-115, 2015. https://doi.org/10.1016/j.agwat.2014.05.008

\begin{tabular}{lcrrr} 
SEIDEL, & S. J.; & WERISCH, S.; \\
BARFUS, & K.; & WAGNER, & M.; \\
SCHÜTZE, & N.; & LABER, & H. \\
Field evaluation & of & irrigation \\
scheduling & \multicolumn{2}{c}{ strategies } & using a \\
mechanistic & crop & growth & model. \\
Irrigation & and drainage, v. 65, n. 2, \\
p. 214-223, & 2016. & https://doi.org/10.1002/ \\
ird.1942 & &
\end{tabular}

